First publ. in: Journal of Comparative Physiology A: Neuroethology, Sensory, Neural, and Behavioral Physiology; 83 (1973), 2. - pp. 123-134

DOI: $10.1007 / B F 00696891$

\title{
Rotation Compensating Reflexes Independent of the Labyrinth
}

\author{
Neurosensory Correlates in Pigeons
}

\begin{abstract}
Summary. Pigeons without labyrinths or with sectioned spinal cords continue to show compensatory reflexes to passive body rotations. This paper reports electrophysiological efforts to identify the source of afference controlling these reflexes. A proportion of fibres in lumbosacral spinal dorsal roots were found to respond briskly to body rotations around the longitudinal and the transversal axes and less to rotation around the vertical axis. Their responsiveness to rotation was unaffected by sectioning the spinal cord at a rostral level and by cutting the somatic branch of the spinal nerve corresponding to the relevant dorsal root. It could be demonstrated that movements of the viscera brought about in two different ways were adequate stimuli for these "rotation" units. It is surmised that the sense organs responsible are stretch receptors located in the mesenteries. The arrangement of mesenteries is deemed suitable for the detection of visceral movements caused by body rotation. Concordances between the behaviour of the rotation units and responses of muscle units involved in the righting reflexes as described by BiedermanThorson and Thorson (1973) strongly suggest that the afferences described are those controlling the reflexes of labyrinthless or spinally transected pigeons.
\end{abstract}

Zusammenfassung. Tauben ohne Vestibularorgane oder mit durchtrenntem Rückenmark zeigen auf passive Körperdrehungen kompensatorische Reflexe. Die vorliegende Arbeit berichtet über elektrophysiologische Versuche, den Ursprung der verantwortlichen Afferenzen festzustellen. Eine Anzahl Fasern der dorsalen spinalen Wurzeln im lumbosacralen Bereich antworteten empfindlich auf Körperdrehungen um die Längs- und Querachse, weniger ausgeprägt auf Drehungen um die Hochachse. Die Antworten blieben bei rostraler Durchtrennung des Rückenmarks erhalten, ebenso, wenn die somatischen Äste des Spinalnervens durchschnitten wurden. Bewegung der Eingeweide, hervorgerufen durch zweierlei Methoden, erwies sich als ein adäquater Reiz für diese „Körper-Kipp“-Einheiten. Es wird angenommen, daß die Afferenzen aus Streckrezeptoren in den Mesenterien kommen. Die Anordnung der Mesenterien erscheint ausreichend geeignet für die eindeutige Wahrnehmung von durch Kippungen des Körpers hervorgerufenen Eingeweidebewegungen. Übereinstimmung zwischen dem Verhalten der Körper-Kipp-Einheiten und die Reaktionen von reflexbeteiligten Muskeleinheiten, wie sie von Biederman-Thorson und Thorson (1973) beschrieben worden sind, bekräftigen die Vermutung, daß die beschriebenen Afferenzen diejenigen sind, die die Gleichgewichtsreflexe von labyrinthlosen und spinalen Tauben kontrollieren. 


\section{Introduction}

When pigeons are held by the body and tilted or turned in various directions, they perform wing, tail and neck movements that clearly function towards compensating the passive displacements. These righting reflexes have generally been assumed to depend on information originating from the vestibular organs (Ten Cate, 1936). Indeed an ingenious control theory model has been proposed on this basis to account for the reflexes shown by pigeons when subjected to various head and body tilts (Mittelstaedt, 1964). As mentioned by Mittelstaedt, observations made long ago by Singer (1884), Trendelenburg (1906) and others on labyrinthectomized birds and birds with sectioned spinal cords suggest, however, that some of the wing, tail and leg righting responses are independent of vestibular afferences. This has been recently confirmed in a thorough series of experiments by Biederman-Thorson and Thorson (1973) who studied the tail and wing reflexes of normal, labyrinthless and spinally transected pigeons to passive body tilting with myographic techniques. There is no doubt that pigeons have another source of information as to rotation of the body than the vestibular organs. This information appears to enter the spinal cord mainly at lumbar levels but its precise origin is obscure. We have attempted to elucidate this latter question further by recording electrophysiologically from dorsalroot fibres and also though less extensively from dorsal horn cells and dorso-lateral funiculi fibres under various experimental conditions.

We wish to thank Dr. H. Mittelstaedt for his hospitality and his collaborators for the assistance they gave us. We are grateful to Drs. M. Biederman-Thorson and J. Thorson for their friendly and critical help while planning this work and preparing this report.

\section{Methods}

23 domestic pigeons (Columba livia) were anaesthetized with Equithesin (intramuscular, initial dose $0.25 \mathrm{ml} / 100 \mathrm{~g}$, additional doses $0.08 \mathrm{ml} / 100 \mathrm{~g}$ ). Two lateroposterior and one anteromedial dorsal surfaces of the pelvic bone were exposed by resecting the overlying skin. The porous bone was pitted with a dental drill and a specially designed holder was cemented to these three points with dental acrilic. The holder with the attached pigeon could be clamped to a semicardanically suspended, counterweighted turntable such that the abdominal cavity of the pigeon was approximately centered and its attitude was nearly normal. The vertebral canal was opened to expose a length of spinal eord with the corresponding three to four pairs of dorsal roots. The technique allowed stable recording from root 21 through to root 27 (counted from the first cervical root). More anterior roots were not sufficiently anchored for satisfactory recording due to vertebral movement.

Artificial respiration was instituted by intubating the trachea and passing a steady stream of air that escaped through the air sac diverticles that had been opened by the resection of pneumatic bone. The animal's temperature was maintained with the help of a $100 \mathrm{~W}$ lamp. Platinum black tipped tungsten microelectrodes $[\mathrm{Z} 1000 \mathrm{~Hz}) \approx 12 \mathrm{M} \Omega]$ made with conventional methods from $0.01 \mathrm{~mm}$ 
diameter wire ensuring a degree of mechanical flexibility were used. They were advanced with a ball-joint mounted microdrive of our own construction. Potentials were amplified with a turntable mounted, high impedance, capacity compensated preamplifier operating with a passband between 0.1 and $3 \mathrm{kHz}$ and an additional $\tilde{50} \mathrm{~Hz}$ notch filter. The output was monitored with an audio unit and an oscilloscope. A multichannel FM tape-recorder registered the neuro-electric events as well as potentials deriving from potentiometers attached to the turntable axles. These coded the tilting and turning of the table that was produced by hand. When appropriate the tape-recorded data was filmed with an oscilloscope camera.

Microelectrode penetrations were aimed at the dorsal roots centrally from the dorsal root ganglia close to their junction with the spinal cord, where they split up into a number of more or less discrete rootlets. Later sometimes penetrations were aimed successively at more medial portions of the spinal cord itself. As the electrode was advanced through nervous tissue the animal was intermittently tilted over 30 degrees to both side of its normal position around either its longitudinal or its transversal axis. When a unit was identified as responding to this stimulation its reaction to stepwise changes of pitch and roll up to $45^{\circ}$ either side from the normal position was recorded. Each position was retained for at least 10 sec. Next its response, to approximately sinusoidal rotation of 60 degrees amplitude at frequencies varying between 0.1 and $2 \mathrm{~Hz}$ around all the body axes (longitudinal, transversal, vertical) was successively investigated. In some cases tilting around a non-standard axis was also tried. Finally we attempted investigating tilts of more than $45^{\circ}$ from the normal position. Many units however were lost before the complete testing regime had been administered.

\section{Results}

Results of dorsal root recordings, to which we directed our main effort, are presented first. An appreciable proportion of fibres there were found to be responsive to pitch and roll changes, fewer to jaw motions. None responded to manual rotation of the head alone and the proportion of responsive units was not noticeably affected by section of the spinal cord at a cephalad level. This indicated that the units were not indirectly driven by vestibular outputs. Nonetheless the spinal cord was routinely cut in all further experiments. Quite a few units however were clearly driven by somatic afferences, probably by cutaneous and joint receptors, when feathers and extremities were passively displaced by the body rotation. This was facilitated by the loss of muscular tonus due to the deep anaesthesia, and it is highly unlikely to be important in the release of righting reflexes. Slight changes of position of a leg or a feather for example often abolished the tilt sensitivity of such units and BiedermanThorson and Thorson (1973) showed that myographic righting reflexes persist unaffected in tightly constrained subjects where the movement of appendages was severely restricted. To exclude these incidentally responsive units the somatic, mixed nerve branches corresponding to the dorsal roots being examined were cut distally through trephinations of the sacral bone some $3-5 \mathrm{~mm}$ lateral from the midline in all final $13 \mathrm{ex}$ periments. This left the dorsal roots with the rami communicantes, 


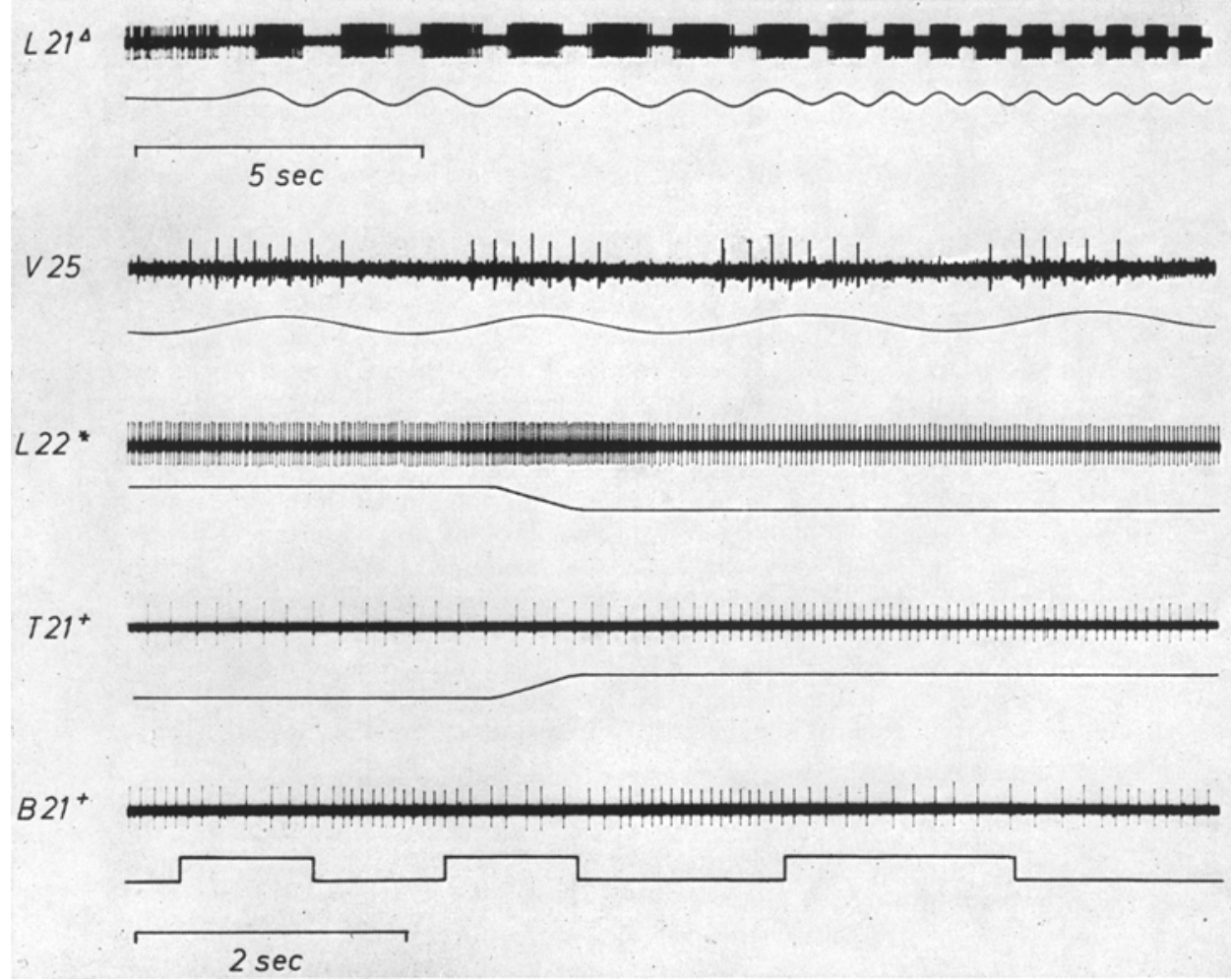

Fig. 1. Oscillographic recordings of responses of pigeon dorsal root units to nearly sinusoidal (two upper recording), nearly step (two middle recordings) body rotations and to intraperitoneal balloon inflation (bottom recording). The spinal cord was transected at a level cephalad from the relevant dorsal root and the somatic nerve branch corresponding to it cut distally. The letters $L, T^{\prime}$ and $V$ refer to the longitudinal, transversal and vertical axis about which the birds body was rotated, $B$ refers to the balloon experiment. The numbers denote the dorsal root (counted through from the first cervical root) where the unit was found. The asterisk, triangle and cross identify individual units for the two first of which further data is presented in Fig. 2 and 3. Note that one time scale applies to the upper recording and another to the four lower recordings. The lower traces of the four upper recordings are a potentiometer records of the body rotations where an upward deflection signifies, depending on the axis, an ipsilateral side upward, a head downward, or a head towards ipsilateral rotation. The amplitude of the sinusoidal rotation was approximately 60 degrees, that of the step tilts 90 degrees. The lower trace of the bottom recording is a contact record where an upward deflection indicates inflation of the balloon. The volume of the balloon of course lagged somewhat with respect to the inflation 
originating in the ipsilateral sympathetic chains, as the only sources of afference. No units could then be found that responded to somatic stimulation, but there was no difficulty in identifying, now often single, units that responded to body rotation (Fig. 1).

32 of these units could be investigated in some detail All roots examined contained responsive units. Those studied intensively were distributed as follows: root 21,3 units; root 22,8 units; root 23,1 unit; root 24,4 units; root 25,4 units ; root 26,10 units; root 27,2 units. This suggests that within the roots examined the afferences signalling rotation preferentially reach the spinal cord through roots 22 and 26 . However, we are not completely satisfied that our sampling was suitably even. Recordings from anterior roots up to root 18 though not totally satisfactory, suggested that similar units might be present at these levels as well.

31 of the intensively studied units fired spontaneously at rates varying between 2 and 75 spikes per sec when the pigeons were in the normal attitude. One unit did not show such behaviour and for this unit and three others with very low baseline firing rates, later statements about activity inhibition must be disregarded. Units with no spontaneous resting discharge may well be under-represented in the sample, they were simply more difficult to detect.

Except for one unit responsive only to pitch changes all 31 remaining units were responsive to rotation both around the transversal and the longitudinal axis. In the pitch mode 19 units increased their firing rate with head downwards motions and decreased it with head upward motions. 12 units reacted in the opposite way. The predominance of the former type of responding units was due to those recorded from the more caudal roots; units from the more rostral roots distributed themselves equally among the two response types. In the roll mode the firing rate of 16 units accelerated with an ipsilateral side upwards motion and decelerated with an ipsilateral side downwards rotation. 13 units responded in the opposite way and one unit's activity was inhibited by rotation to either side. Only 15 of the 32 units respond to yaw accelerations, some of them rather weakly. 9 of them were activated by turning towards the contralateral side and inhibited by turning towards the ipsilateral side, 6 responded in the opposite way. The way in which a given unit responded to rotation around one axis did not allow one to predict how it would respond to tilting around another: all possible response type combinations occurred.

45 or 90 degrees "step" tilts at approximately 180 degrees per sec revealed an extremely varied spectrum of response dynamics that defies any simple classification. Some samples are given in Fig. 2. The dynamic response pattern of a given unit however was closely reproduceable, at 


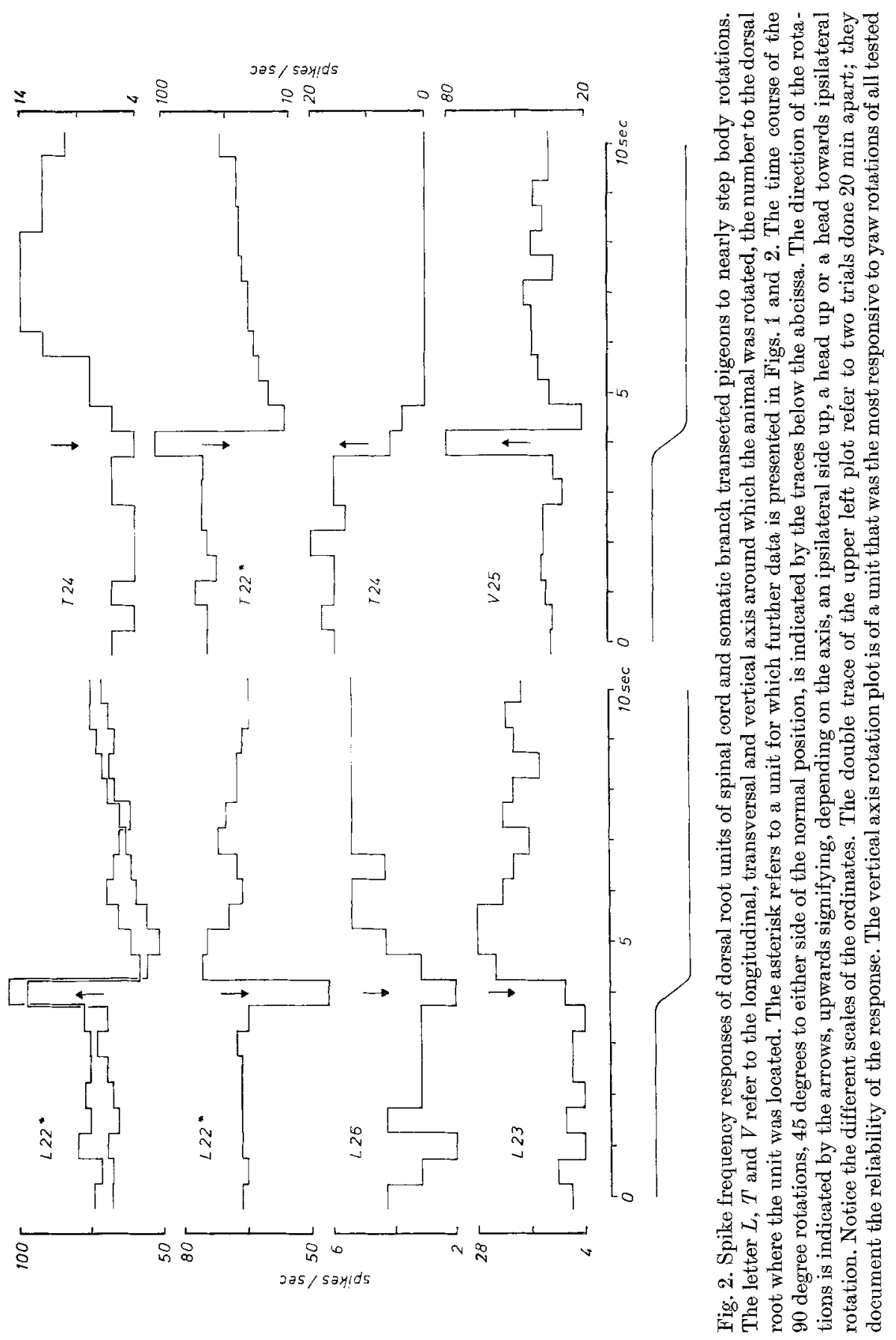


least when tested up to $20 \mathrm{~min}$ apart. Some units may have responded tonically to given static tilt but the activity of 3 such units that were carefully examined in this respect did eventually adapt or drift after several minutes. The response characteristics of a given unit to such "step" tilts tended to be similar for both the pitch and roll modes, the main differences seem to be in terms of amplitude. Responses to yaw "steps" however, if detectable, were invariably transient and almost always of considerably less amplitude.

5 units could be exposed to approximately sinusoidal rotation over a range of frequencies and about $60^{\circ}$ amplitude around all three axes. Samples of some units' response to this type of stimulation are shown in Fig. 3. Even allowing for the imperfection of the input sinusoids there can be little doubt about the individually varying non-linear behaviour of most units. For roll and pitch the gain of all 5 units diminished as the input frequency decreased below about $2 \mathrm{~Hz}$ and 2 units probably also showed a decrease of gain at the highest frequencies that we were able to produce $(4 \mathrm{~Hz}$, appreciable deviation from true sines and only about $30^{\circ}$ amplitude). At the lowest frequencies phase shifts approaching 0 and 180 degrees were observed. As the frequency increased to about $2 \mathrm{~Hz}$ the phase shifts changed concordantly to values of +60 to +90 and of -90 to -120 degrees. At even higher frequencies phase shifts were uncertain due to the small amplitude of the responses. At the same frequencies of sinusoidal pitch and roll stimulation the responses of a given unit tended to be similar although some differences in gain, phase and non-linearity did clearly maintain. Equivalent sinusoidal yaw rotations showed that if units responded at all, the maximum gain lay at the highest frequencies attainable (here about $5 \mathrm{~Hz}$ ) and that it decreased rapidly towards the lower frequencies: with a $0.1 \mathrm{~Hz}$ input no response could be detected in any of the units. Phase shifts ranged widely and no pattern could be discerned.

Tilting with respect to the longitudinal and transversal axes at mean body positions up to 180 degrees different from the normal led to loss of contact with most units found while the bird was in the natural position. It was technically difficult to search for new ones at these positions in our apparatus; we were thus only able to ascertain the presence of rotation units responding at these angles but not to study their behaviour in any detail. Rotations around various non standard axes that were tried with some units sometimes yielded an axis that resulted in markedly larger response gains than those around the normal axes. However, we did not explore this in a systematic enough way.

The activity of 5 out of 6 rotation sensitive fibres proved to be modulated by normal respiration when animals were allowed to reassume it in two experiments. Intermittent inflation of the lung-airsac system by 

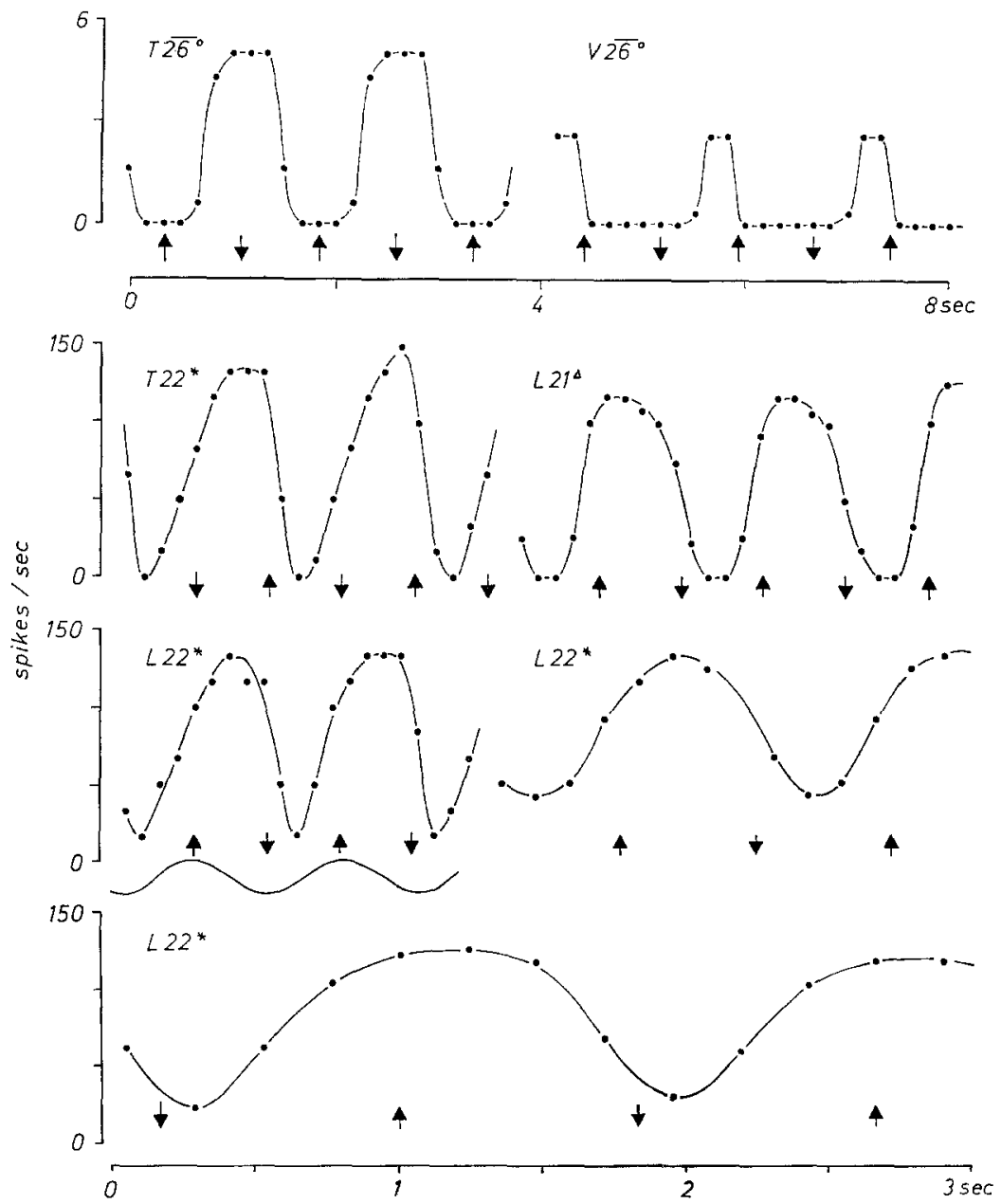

Fig. 3. Spike frequency responses of dorsal root units of spinal cord and somatic branch transected pigeons to nearly sinusoidal body rotations of various frequencies. The letter $L, T$ and $V$ refer to the longitudinal, transversal and vertical axis around which the animal was rotated, the number to the dorsal root where the unit was located. The asterisk, triangle and circle refer to individual units, for the two first of which further data is presented in Fig. 1 and 2. Bars indicate that the plots are repeated averages from 3 cycles. The sinusoidal rotations had an amplitude of approximately 60 degrees, 30 degrees to either side of the normal position. A sample timecourse of such rotation is given with one of the plots. Upwards arrows indicate, depending on the axis, a peak rotation ipsilateral side up, head up or head towards ipsilateral. Notice the different scales of the abseissas and the ordinates 
occlusion of the artificial respiration airstream outlet in the same experiments did also drive 3 rotation units but less effectively so than normal respiration. We did find however, a few units that responded briskly to this treatment but they were not sensitive to body tilting. Two rotation units also responded with heartbeat (electrocardiogram) locked brief frequency changes. Occasionally, the activity of rotation units was temporarily affected by irregular changes. This could well have been due to bursts of gastrointestinal motility as observed during laparotomies. Slower but sometimes marked variations in baseline activity of uncertain origin were noticed in 4 out of 6 units that were held for more than $30 \mathrm{~min}$.

Several days before dorsal root recording two birds were equipped, after laparotomy, with a balloon between the viscera and the body wall with an externalized mouth. Intermittent inflation of these balloons with 10 to 25 cc of air invariably was effective in either activating ( 2 units) or inhibiting (4 units) 6 units that were also responsive to body rotation (Fig. 1). Two further birds were equipped, again by laparotomy, but immediately before recording, with a stiff wire hook that was carefully inserted among the viscera and had an external handle. All 5 rotation sensitive units tested could again, without exception be activated or inhibited by gentle movement of this hook. In both types of operations we failed to find any units that would respond to the manipulation of the viscera but which would not respond to body rotation. However, our search for such fibres was perhaps not sufficiently intensive.

Additionally to the dorsal root units we recorded from a number of presumed, i.e. inferred from electrode position with no histological controls, dorsal horn cells and dorso-lateral funiculus fibres that similarly responded to body rotation. While it was relatively easy to find responsive dorsal horn cells-there was some evidence that they clustered in nuclei slightly caudally from each dorsal root entry - difficulties arose from their electrode pressure sensitivity. A proportion of the cells suspected to be truly responsive to body rotation did also respond to electrode pressure changes brought about by microdrive adjustments. Since electrode position changes, seen as variations in spike amplitude, were often concommittants of body rotations, there was a lingering uncertainty as to the source of the spike frequency changes observed with tilting in these cells. Recordings from only 5 cells were deemed to be free from this effect.

Rotation-responsive dorso-lateral funiculus fibres were virtually insensitive to electrode pressure but were relatively difficult to find and to hold. Sufficiently extensive recordings are only available for 3 units. These 8 spinal units showed a behaviour that was closely similar to that of dorsal root rotation units except that all units of this small sample had high resting discharges and showed fast and complete adaptation to step tilts i.e. had a small gain at low stimulus frequencies. They probably 
were also less prone to drift than the root units. It is possible that their activity reflected the integration of afferences through several dorsal root units: 2 spinal units that were tested in the visceral hook experiments responded to the stimulation but seemed to be considerably more particular about the precise visceral motion than the rather unselective dorsal root units.

\section{Discussion}

We conclude that these afferences originating from within the peritoneal cavity could be those controlling the righting reflexes of labyrinthless or spinally transected pigeons described by previous authors. Within the range of rotational stimulation that we studied the behaviour of at least some of the relevant dorsal root units was consonant with behaviour of units from righting reflex supporting muscles that Biederman-Thorson and Thorson (1973) describe, i.e. they did not exhibit d.c. responses to static tilts and at frequencies of 0.5 cycles per sec sinusoidal rotations around the normal body position they showed phase shifts of approximately +45 or -135 degrees. We were unable to secure our preparation rigidly enough to see whether the correspondence would hold for sinusoidal stimulation at mean body positions other than the normal. This would have been helpful insofar that the Thorson's found the mean body position to affect the gain and phase of the righting reflexes in a characteristic manner. Our finding that the rotation units were largely insensitive to yaw motions, i.e. to purely inertial stimulation, corresponds with the Thorsons' finding that the righting reflexes do not depend primarily on angular body accelerations. The observation that tilting around a particular non-standard axis was sometimes a more effective stimulus for our units than rotation around a standard one also concords with Biederman-'Thorson and Thorson's (1973) descriptions.

Based on the finding that the dorsal root units in question could be driven by visceral movements implemented with the intraperitoneal balloons and hooks we surmise further that, as already Singer (1884) and Trendelenburg (1906) speculated with reference to the righting reflexes, the afferences originate from mechanoreceptors located in mesenteries. In thick body sections prepared by sawing frozen pigeons in various planes we found that the mesenteries have multiple, radial attachments to the peritoneal walls. This arrangement would seem to be adequate for the detection of motions of the viscera relative to the body as they occur during body rotations given that they are furnished with suitable stretch receptors ${ }^{1}$. Such receptors are known to exist in mammals (Leek, 1971)

1 The close packing of organs in the pleural cavity makes it less likely as a substrate for a rotation detecting system. 
but have yet to be demonstrated in birds. The sympathetic innervation of the avian viscera is however at least as extensive as that of mammals. Birds actually possess, in addition to the splanchnic and coeliac ganglion, another major, string-like mesenterial plexus that is not present in mammals: the nervus intestinalis (Hsieh, 1951).

There is, in this context, a divergence between our and BiedermanThorson and Thorson's (1973) balloon experiment results that requires explanation. They could not elicit myographic reflexes with visceral displacement brought about by intraperitoneal balloons. The disparity, we think, may be due to the fact that the reflex activity of the muscles is driven by a neural outflow that is probably the result of the integration of the afferences of a large number of dorsal root "rotation" units entering the spinal cord over several segments. Balloon inflation as we could observe during laparotomies did not displace the viscera in the same concerted way as body tilting did, rather it produced local displacements often in opposite directions. We think therefore that balloon inflation was ineffective as a reflexogenic stimulus because it failed to produce afferences with the necessary coherence. The Thorsons' also found that intraperitoneal injections of Novocain were ineffective in abolishing the righting reflexes. Similarly, we found that they did not suppress the rotation responsiveness of our units. However, control injections of comparable amounts of indian ink followed by laparotomy showed that such injections do not reach more than a minute portion of the peritoneal volume. Mesenterial membranes prevent any effective perfusion. We can not however explain why the 'Thorsons' failed to elicit compensatory tail reflexes in a pigeon with a spinal cord transection just anterior to root 24 in view of the fact that we found rotation units in dorsal roots up to at least root 27 . It may be that afferences through only few roots are just not sufficient to drive the corresponding motorneurons.

Objections that the visceral-mesenterial stretch receptor system would be an inadequate gravital-inertial sensing device because of the varying weight of the gut content are not justified. The frequency spectrum of the load changes should be well below the range of frequencies where the righting reflexes show an appreciable gain. Gastric motility disturbances which probably have a frequency spectrum closer to this range could be ineffective because of their locally disjunct character. This must produce afferences with a coherence quite different from that produced by body rotation, thus allowing suppression by neural filtering. The respiratory modulation of the rotation units' activity may be dealt with in a similar way. In any case, it is difficult to see what other structures could provide the sensory information to mediate the righting reflexes of spinally transected pigeons. 
We may finally wonder why at least some birds have evolved a body posture controlling mechanism additional to vestibularly driven reflexes. The answer probably lies in the fact that arboreal and aerial birds require particularly fast righting reflexes. The compensation for neck torsion that vestibular information must undergo to be useful for body orientation control (Mittelstaedt, 1964)-particularly in animals with a neck as mobile as that of birds-may well introduce incompatible delays. The "visceral" reflexes presumably function to provide an initial, rapid, even if possibly gross, compensation before vestibular reflexes provide the fine, though slow correction of passive body rotations.

\title{
References
}

Biederman-Thorson, M., Thorson, J.: Rotation-compensating reflexes independent of the labyrinth: neuromuscular correlates in the pigeon. J. comp. Physiol., 83, 103-122 (1973).

Hsieh, T. M.: The sympathetic and parasympathetic nervous system of the fowl. $\mathrm{Ph}, \mathrm{D}$. Thesis, Edinburgh 1951.

Leek, B. F.: Abdominal visceral receptors. In: Autrum, H., et al. (eds.), Handbook of sensory physiology, vol. 3 (1), p. 113-160. Berlin-Heidelberg-New York: Springer 1972.

Mittelstaedt, H.: Basic control patterns of orientational homeostasis. Symp. Soc. exp. Biol. 18, 365-385 (1964).

Singer, S.: Zur Kenntnis der motorischen Funktionen des Lendenmarks der Taube. S.-B. Akad. Wiss. Wien, math.-nat. Kl. 89, 167-185 (1884).

Trendelenburg, W.: Weitere Untersuchungen über die Bewegungen der Vögel nach Durchschneidung der Rückenmarkwurzeln. Arch. Physiol. 1906, Suppl., 231-245 (1906).

Ten Cate, J.: Physiologie des Zentralnervensystems der Vögel. Ergebn. Biol. 13 93-173 (1936).

\author{
Dr. Juan D. Delius \\ Department of Psychology \\ University of Durham \\ Science Laboratories, South Road \\ Durham, England \\ Fritz W. Vollrath \\ Zoologisches Institut \\ Universität Freiburg i. Br. \\ Katharinenstr. 20 \\ D-7800 Freiburg i. Br. \\ Federal Republic of Germany
}

